Pacific Journal of Mathematics

TOPOLOGIES ON THE QUOTIENT FIELD OF A DEDEKIND 


\title{
TOPOLOGIES ON THE QUOTIENT FIELD OF A DEDEKIND DOMAIN
}

\author{
JO-ANN COHEN
}

\begin{abstract}
It is well known that if $D$ is a Dedekind domain with quotient field $K$ and if $T$ is any Hausdorff nondiscrete field topology on $K$ for which the open $D$-submodules of $K$ form a fundamental system of neighborhoods of zero, then $T$ is the supremum of a family of $p$-adic topologies. We show that if the class number of $K$ over $D$ is finite and if $T$ is any Hausdorff nondiscrete field topology on $K$ for which $D$ is a bounded set, then $T$ is the supremum of a family of $p$-adic topologies. We then investigate the problem of extending a locally bounded topology from $D$ to a locally bounded topology on $K$. The extendable topologies on $D$ for which there exists a nonzero topological nilpotent and for which $D$ is a bounded set are characterized. Moreover it is shown that the topology of a locally compact principal ideal domain $A$ extends to a ring topology on the quotient field of $A$ if and only if $A$ is compact.
\end{abstract}

1. Introduction and basic definitions. Let $R$ be a commutative ring and let $T$ be a ring topology on $R$, that is, $T$ is a topology on $R$ making $(x, y) \rightarrow x-y$ and $(x, y) \rightarrow x y$ continuous from $R \times R$ to $R$. A subset $A$ of $R$ is bounded for $T$ if given any neighborhood $U$ of zero, there exists a neighborhood $V$ of zero such that $A V \subseteq U . T$ is a locally bounded topology on $R$ if there exists a fundamental system of neighborhoods of zero for $T$ consisting of bounded sets. As every compact set is bounded [4, Exercise 12 , p. 119], if $T$ is a ring topology on $R$ and $(R, T)$ is locally compact, then $T$ is a locally bounded topology on $R$.

Each norm $N$ on a ring $R$ defines a locally bounded topology $T_{N}$ on $R$ in a natural way. Obviously, each norm-bounded subset of $R$ is also bounded for $T_{N}$. Furthermore, if $N$ is a nontrivial norm on a field $K$, that is, $T_{N}$ is nondiscrete, then a subset $A$ of $K$ is bounded in norm if and only if $A$ is bounded for $T_{N}$.

Let $D$ be a Dedekind domain that is not a field, let $K$ be the quotient field of $D$ and let $\mathscr{P}$ be the set of nonzero proper prime ideals of $D$. We assume familiarity with the definitions and basic properties of the functions $n_{p}$ defined on the set of nonzero fractionary ideals of $K$ and the valuations $v_{p}$ defined on $K$ for each $p$ in $\mathscr{P}$. (See for example [3, pp. 25-26].) If $F$ is a field and $x$ is a transcendental element over $F$, we denote the valuation on $F(x)$ defined by the prime ideal $\left(x^{-1}\right)$ of $F\left[x^{-1}\right]$ by $v_{\infty}$. 
In [9], Correl proved that if $T$ is any nondiscrete Hausdorff field topology on the quotient field $K$ of a principal ideal domain $D$ for which the open $D$-submodules of $K$ form a fundamental system of neighborhoods of zero, then $T$ is the supremum of a family of $p$-adic topologies. Jebli showed that this characterization also holds if $D$ is a Dedekind domain [14]. Heine and Warner gave a further generalization in [13]. In $\S 2$, we show that if $K$ is the quotient field of a Dedekind domain $D$ such that the class number of $K$ over $D$ is finite and if $T$ is any nondiscrete Hausdorff field topology on $K$ for which $D$ is a bounded set, then $T$ is the supremum of a family of $p$-adic topologies. This result also yields known results concerning norms on algebraic function fields [6, Theorems 1,2 and 18, Theorem 2.11].

The problem of extending ring topologies from an integral domain $I$ to its quotient field $F$ has been widely considered. Gelbaum, Kalisch and Olmsted gave sufficient conditions for which $F$ possesses a field topology whose restriction to $I$ is weaker than the original topology [11]. These results were extended by Endo in [10], but again the restriction to $I$ of the topology on $F$ is in general weaker than the given topology. In [1], Anthony gave necessary and sufficient conditions for the topology on $I$ to be the restriction of a certain topology defined on $F$. However, the topology on $F$ is not necessarily compatible with the ring structure of $F$. Other general results on the extension problem can be found in [2].

In $\$ 3$ of this paper, we give criteria for which there exists a locally bounded topology on the quotient field $K$ of a Dedekind domain $D$ whose restriction to $D$ is a given locally bounded topology. We also characterize all Hausdorff, nondiscrete, extendable locally bounded topologies on $D$ for which $D$ is a bounded set and for which there exists a nonzero topological nilpotent in $D$ (that is, a nonzero element $c$ in $D$ such that $c^{n} \rightarrow 0$ ) when the class number of $K$ over $D$ is finite. Then in $\S 4$ we consider the problem of extending locally compact topologies from a Dedekind domain to its quotient field.

\section{Field topologies on the quotient field of a Dedekind domain.} Throughout this section let $D$ be a Dedekind domain which is not a field, let $K$ be the quotient field of $D$, let $\mathscr{P}$ denote the set of nonzero proper prime ideals of $D$ and for each $p$ in $\mathscr{P}$, let $T_{p}$ denote the locally bounded topology on $K$ defined by the valuation $v_{p}$.

LeMma. Suppose the class number $\alpha$ of $K$ over $D$ is finite. Let $y_{1}, y_{2}, \ldots, y_{\alpha}$ be nonzero elements of $K$, let $p_{1}, p_{2}, \ldots, p_{n}$ be distinct elements 
of $\mathscr{P}$ and let $M$ be any positive integer. If $v_{p_{i}}\left(y_{j}\right) \geq M$ for all $i$ in $[1, n]$ and all $j$ in $[1, \alpha]$, then there exist nonzero elements $a$ and $b$ in $D$ such that $y_{1} y_{2} \cdots y_{\alpha}=a / b$ and $v_{p_{t}}(a), v_{p_{i}}(b-1) \geq M$ for all $i$ in $[1, n]$.

Proof. For each $j$ in $[1, \alpha]$, let $A_{j}$ and $B_{j}$ be nonzero ideals of $D$ such that $D y_{j}=A_{j} B_{j}^{-1}$ and $A_{j}+B_{j}=D$. Clearly $n_{p_{t}}\left(A_{j}\right) \geq M$ for all $i$ in $[1, n]$ and all $j$ in $[1, \alpha]$. Let $A=\sum_{j=1}^{\alpha} A_{j}$ and let $B=\bigcap_{j=1}^{\alpha} B_{j}$. Then $A$ and $B$ are nonzero ideals of $D$ such that $A+B=D$. Indeed, suppose $p$ in $\mathscr{P}$ is such that $n_{p}(A) \geq 1$. Then $n_{p}\left(A_{j}\right) \geq 1$ for all $j$ and hence $n_{p}\left(B_{j}\right)=0$ for all $j$. Thus $n_{p}(B)=0$ and so $A+B=D$. Therefore, $A^{\alpha}+B^{\alpha}=D$ as well. Since $\alpha$ is the class number of $K$ over $D$, there exist nonzero elements $c$ and $d$ in $D$ such that $A^{\alpha}=D c$ and $B^{\alpha}=D d$. We note that for all $i$ in $[1, n], v_{p_{t}}(c)=n_{p_{i}}\left(A^{\alpha}\right)=\alpha \min \left\{n_{p_{t}}\left(A_{j}\right): 1 \leq j \leq \alpha\right\} \geq \alpha M \geq M$. In particular, $c$ is a nonunit of $D$. As $A^{\alpha}+B^{\alpha}=D$, there exist elements $s$ and $t$ in $D$ such that $1=s c+t d$. By the above remarks, $t \neq 0$ and $v_{p_{i}}(t d-1) \geq$ $M$ for all $i$ in $[1, n]$. Therefore in order to complete the proof of the lemma, it suffices to show that there exists a nonzero element $x$ in $D$ such that $y_{1} y_{2} \cdots y_{\alpha}=x c / t d$.

By the definitions of $A$ and $B, A_{j} \subseteq A$ and $B \subseteq B_{j}$ for all $j$ in $[1, \alpha]$. Hence

$$
y_{1} y_{2} \cdots y_{\alpha} \in\left(A_{1} A_{2} \cdots A_{\alpha}\right)\left(B_{1}^{-1} B_{2}^{-1} \cdots B_{\alpha}^{-1}\right) \subseteq A^{\alpha}\left(B^{-1}\right)^{\alpha}=D \frac{c}{d} .
$$

So there exists a nonzero $y$ in $D$ with $y_{1} y_{2} \cdots y_{\alpha}=y c / d$. Then $y_{1} y_{2} \cdots y_{\alpha}$ $=x c / t d$ where $x=t y$.

THEOREM 1. Let $D$ be a Dedekind domain which is not a field, let $K$ be the quotient field of $D$ and let $\mathscr{P}$ be the set of nonzero proper prime ideals of $D$. Suppose the class number $\alpha$ of $K$ over $D$ is finite. If $T$ is a nondiscrete Hausdorff field topology on $K$ for which $D$ is a bounded set, then $T$ is the supremum of a family of p-adic topologies.

Proof. For each $p$ in $\mathscr{P}$, let $B_{p}=\left\{y \in K: v_{p}(y)>0\right\}$. Let $A$ be any $T$-neighborhood of zero. We first show that there exists a nonzero proper ideal $I$ of $D$ such that $I \subseteq A$ and $B_{p}$ is a $T$-neighborhood of zero for each $p$ in $\mathscr{P}$ with $n_{p}(I)>0$. As $T$ is Hausdorff, we may assume that $D$ is not a subset of $A$. Furthermore, we may assume that $A$ is a closed $T$-neighborhood of zero. Let $W=\{y \in K: y D \subseteq A\}$. Clearly, $W \subseteq A$ and $W D \subseteq A$. Moreover as $D$ is a $T$-bounded set, $W$ is a $T$-neighborhood of zero. Since $T$ is nondiscrete, there exist nonzero elements $a$ and $b$ in $D$ such that $a / b \in W$. By the above remarks, $D a=D b(a / b) \subseteq A$. As $D$ is not a 
subset of $A, a$ is a nonunit of $D$. Thus $A$ contains a nonzero proper ideal $I$ of $D$ which we may assume is closed for $T$. Let $p_{1}, p_{2}, \ldots, p_{n}$ be distinct elements of $\mathscr{P}$ and let $\alpha_{1}, \alpha_{2}, \ldots, \alpha_{n}$ be positive integers such that $I=$ $\prod_{i=1}^{n} p_{i}^{\alpha_{i}}$. Suppose that there exists an $i$ such that $B_{p_{i}}$ is not a $T$-neighborhood of zero. Without loss of generality, assume that $i=1$. As $I$ is $T$-closed, $I=\bigcap\{V+I: V$ is a $T$-neighborhood of zero $\}$. Let $V$ be any $T$-neighborhood of zero and let $V_{1}=\{y \in K: y D \subseteq V\}$. As before $V_{1}$ is a $T$-neighborhood of zero, $V_{1} \subseteq V$ and $D V_{1} \subseteq V$. By assumption, there exist nonzero elements $c$ and $d$ in $D$ such that $v_{p_{1}}(c / d) \leq 0$ and $c / d \in V_{1}$. Let $x$ be a nonzero element of $D$ such that $v_{p_{1}}(x)=-v_{p_{1}}(c / d)$ and $v_{p}(x)=-v_{p}(c / d)$ for all $p$ in $\mathscr{P}$ with $v_{p}(c / d)<0$. (The existence of $x$ is guaranteed by [3, Proposition 9, p. 12].) Then $x c / d$ is a nonzero element of $D, D x c / d \subseteq V$ and $v_{p_{1}}(x c / d)=0$. Hence $n_{p_{1}}(D x c / d)=0$. Consequently, if $p \in \mathscr{P} \backslash\left\{p_{2}, \ldots, p_{n}\right\}$, then $n_{p}(D x c / d+I)=$ $\min \left\{n_{p}(D x c / d), n_{p}(I)\right\}=0$. Moreover, if $p \in\left\{p_{2}, \ldots, p_{n}\right\}$, then $0<$ $n_{p}(D x c / d+I) \leq n_{p}(I)$. So $D x c / d+I=\prod_{i=2}^{n} p_{i}^{\beta_{i}}$ where $0 \leq \beta_{i} \leq \alpha_{i}$ for $i=2,3, \ldots, n$. Consequently for each $T$-neighborhood $V$ of zero, $\prod_{i=2}^{n} p_{i}^{\alpha_{i}} \subseteq V+I$. Then $\prod_{i=2}^{n} p_{i}^{\alpha_{i}} \subseteq I=\prod_{i=1}^{n} p_{i}^{\alpha_{i}}$, a contradiction. Therefore $B_{p}$ is a $T$-neighborhood of zero for each $p$ in $\mathscr{P}$ such that $n_{p}(I)>0$.

Let $A$ be any $T$-neighborhood of zero. We next show that there exist $p_{1}, p_{2}, \ldots, p_{n}$ in $\mathscr{P}$ and a neighborhood $B$ of zero for $\sup _{1 \leq i \leq n} T_{p_{i}}$ such that $B \subseteq A$ and for all $i$ in $[1, n], B_{p_{t}}$ is a $T$-neighborhood of zero. As $T$ is a field topology on $K$, there exists a $T$-neighborhood $V$ of zero such that $V(1+V)^{-1} \subseteq A$. By the above, there exist distinct elements $p_{1}, p_{2}, \ldots, p_{n}$ of $\mathscr{P}$ and positive integers $\alpha_{1}, \alpha_{2}, \ldots, \alpha_{n}$ such that $B_{p_{t}}$ is a $T$-neighborhood of zero for $i=1,2, \ldots, n$ and $\prod_{i=1}^{n} p_{i}^{\alpha_{i}} \subseteq V$. Then $\Pi_{i=1}^{n} p_{i}^{M} \subseteq V$ where $M=\max \left\{\alpha_{i}: 1 \leq i \leq n\right\}$. Let $B_{1}=\left\{y \in K: v_{p_{i}}(y) \geq M\right.$ for all $i$ in $[1, n]\}$ and let $y_{1}, y_{2}, \ldots, y_{\alpha-1}$ be fixed nonzero elements of $B_{1}$. If $y$ is any nonzero element of $B_{1}$, then by the preceding lemma, there exist nonzero elements $a$ and $b$ in $D$ such that $y_{1} y_{2} \cdots y_{\alpha-1} y=a / b$ and $a, b-1 \in$ $\Pi_{i=1}^{n} p_{i}^{M} \subseteq V$. So $y_{1} y_{2} \cdots y_{\alpha-1} y \in V(1+V)^{-1} \subseteq A$ and hence

$$
y_{1} y_{2} \cdots y_{\alpha-1} B_{1} \subseteq A \text {. }
$$

Therefore the elements $p_{1}, p_{2}, \ldots, p_{n}$ of $\mathscr{P}$ and the set $B$ defined by, $B=y_{1} y_{2} \cdots y_{\alpha-1} B_{1}$, satisfy the desired properties.

Now let $S=\left\{p \in \mathscr{P}: B_{p}\right.$ is a $T$-neighborhood of zero $\}$. By the above remarks, $T \subseteq \sup _{s \in S} T_{s}$. Moreover $\sup _{s \in S} T_{s} \subseteq T$. Indeed, if $\left\{p_{1}, p_{2}, \ldots, p_{n}\right\} \subseteq S$ and $M$ is any nonnegative integer, then $\bigcap_{i=1}^{n} B_{p_{i}}^{M}$ is a $T$-neighborhood of zero contained in $\left\{y \in K: v_{p_{i}}(y) \geq M\right.$ for $i=$ $1,2, \ldots, n\}$. 
COROllaRY. Let $A$ be a principal ideal domain which is not a field and let $K$ be the quotient field of $A$. If $T$ is a nondiscrete Hausdorff field topology on $K$ for which $A$ is bounded, then $T$ is the supremum of a family of $p$-adic topologies.

Corollary 2 (Correl [9]). Let $D$ be a Dedekind domain which is not a field and let $K$ be the quotient field of $D$. Suppose that the class number of $K$ over $D$ is finite. If $T$ is any nondiscrete Hausdorff field topology on $K$ for which the open $D$-submodules of $K$ form a fundamental system of neighborhoods of zero, then $T$ is the supremum of a family of p-adic topologies.

TheOREM 2. Let $D$ be a Dedekind domain which is not a field, let $K$ be the quotient field of $D$ and let $\mathscr{P}$ be the set of nonzero proper prime ideals of $D$. Suppose that the class number of $K$ over $D$ is finite. If $T$ is a Hausdorff ring topology on $K$, then the following are equivalent.

$1^{\circ} T$ is a field topology on $K, D$ is a bounded set for $T$ and there exists a nonzero topological nilpotent for $T$.

$2^{\circ}$ There exists a finite subset $\left\{p_{1}, p_{2}, \ldots, p_{n}\right\}$ of $\mathscr{P}$ such that $T=$ $\sup _{1 \leq i \leq n} T_{p_{i}}$.

$3^{\circ} T$ is a locally bounded topology on $K$, there exists a nonzero topological nilpotent for $T$ and $D$ is a bounded set for $T$.

$4^{\circ} D$ is a bounded set for $T$ and there exists a nontrivial norm on $K$ which defines $T$.

Proof. Suppose $T$ is a field topology on $K, D$ is a bounded set for $T$ and $y$ is a nonzero topological nilpotent for $T$. By Theorem 1 there exists a nonempty subset $S$ of $\mathscr{P}$ such that $T=\sup _{s \in S} T_{s}$. If $S$ is infinite, let $p \in S$ be such that $v_{p}(y)=0$. Then $\left\{z \in K: v_{p}(z)>0\right\}$ is a $T$-neighborhood of zero but $y^{m} \notin\left\{z \in K: v_{p}(z)>0\right\}$ for any $m$, a contradiction. Hence $S$ is finite and so $1^{\circ} \Rightarrow 2^{\circ}$. Clearly $2^{\circ} \Rightarrow 3^{\circ}$. By [8, Theorem 6.1], $3^{\circ} \Rightarrow 4^{\circ}$. The proof that $4^{\circ} \Rightarrow 1^{\circ}$ is the same as that for normed algebras found on page 75 of [5].

Corollary 1. Let $F$ be a field, let $x$ be a transcendental element over $F$ and let $T$ be a Hausdorff locally bounded topology on $F(x)$ for which $F$ is $a$ bounded set. If there exists a nonzero topological nilpotent $f(x)$ in $F[x]$, then $T$ is the supremum of a finite family of p-adic topologies.

Proof. By [8, Theorem 6.1], there exists a nontrivial norm $N$ on $F(x)$ such that $T=T_{N}$. As $F$ is a $T$-bounded set, there exists $M^{\prime}>0$ such that 
$N(a) \leq M^{\prime}$ for all $a$ in $F$. We note that as $T$ is Hausdorff and $F$ is a $T$-bounded set, $f(x) \notin F$. Let $m>0$ be such that $N\left(f^{m}\right)<1$ and let $g=f^{m}$. By the above remarks, $N(g)<1, g \notin F$ and there exists $M>0$ such that $N(h) \leq M$ for all $h$ in $\{t \in F[x]: t=0$ or $\operatorname{deg} t<\operatorname{deg} g\}$. For $y$ in $F[x]$, let $y_{0}, y_{1}, \ldots, y_{n}$ be elements of $F[x]$ such that $y=\sum_{i=0}^{n} y_{i} g^{i}$ and for all $i, y_{i}=0$ or deg $y_{i}<\operatorname{deg} g$. Then

$$
N(y) \leq \sum_{i=0}^{n} N\left(y_{i}\right) N(g)^{i} \leq M \frac{1}{1-N(g)} .
$$

Hence $F[x]$ is bounded in norm and so the corollary follows from Theorem 2.

Corollary $2[6,18]$. Let $F$ be a field and let $x$ be a transcendental element over $F$. If $T$ is a Hausdorff locally bounded topology on $F(x)$ for which $F$ is bounded and for which there exists a nonzero topological nilpotent, then there exists a finite subset $\left\{p_{1}, p_{2}, \ldots, p_{n}\right\}$ of $\mathscr{P} \cup\{\infty\}$ such that $T=\sup _{1 \leq i \leq n} T_{p_{i}}$.

Proof. As before, there exists a nontrivial norm $N$ on $K$ such that $T=T_{N}$. Let $y$ be a nonzero element of $F(x)$ such that $N(y)<1$. As $F$ is a $T$-bounded set, $y \notin F$. So $y$ is a transcendental element over $F, F(x)$ is a finite algebraic extension of $F(y)$ and $\left.T\right|_{F(y)}$ is defined by $\left.N\right|_{F(y)}$. Consequently, $F$ is a bounded subset for $\left.T\right|_{F(y)}$. So by Corollary 1 , there exist nontrivial valuations $v_{1}, v_{2}, \ldots, v_{n}$ on $F(y)$, each of which is trivial on $F$, and corresponding valuation topologies $T_{v_{1}}, T_{v_{2}}, \ldots, T_{v_{n}}$ on $F(y)$ such that $\left.T\right|_{F(y)}=\sup _{1 \leq i \leq n} T_{v_{i}}$. Let $K_{0}$ be a maximal subfield of $F(x)$ containing $F(y)$ such that there exist nontrivial valuations $v_{1}^{\prime}, v_{2}^{\prime}, \ldots, v_{t}^{\prime}$ on $K_{0}$ each of which is trivial on $F$, with $\left.T\right|_{K_{0}}=\sup _{1 \leq i \leq t} T_{v_{i}^{\prime}}$. By Theorem 5 of [21], $K_{0}=K_{1}$ where $K_{1}=\left\{z \in F(x): z\right.$ is separable over $\left.K_{0}\right\}$. If char $F$ $=0$, then $F(x)=K_{1}$. We may therefore assume that char $F=p \neq 0$ and $F(x)$ is a purely inseparable extension of $K_{0}$. So there exists $m \geq 0$ such that $x^{p^{m}} \in K_{0}$. By Theorem 4 of [21] and its proof, for each $i, 1 \leq i \leq t$, there exists a Hausdorff locally bounded topology $T_{i}$ on $F(x)$ such that $\left.T_{i}\right|_{K_{0}}=T_{v_{i}^{\prime}}$ and $T=\sup _{1 \leq i \leq n} T_{i}$. Consequently for each $i$, there exists a nonzero topological nilpotent for $T_{i}$. Thus each $T_{i}$ normable $[8$, Theorem 6.1]. As each nontrivial valuation on $F(x)$ which is trivial on $F$ is equivalent to $v_{s}$ for some $s$ in $\mathscr{P} \cup\{\infty\}$ [3, Corollary 2, p. 94], it suffices to show that each $T_{i}$ is the supremum of finitely many nondiscrete valuation topologies, each of which is discrete on $F$.

Let $1 \leq i \leq t$. Since $v_{i}^{\prime}$ may be extended to a valuation on $F(x)[3$, Proposition 5, p. 105], we may assume that $v_{i}^{\prime}=\left.v_{s}\right|_{K_{0}}$ for some $s$ in 
$\mathscr{P} \cup\{\infty\}$. Suppose $s \in \mathscr{P}$. Then $s=(q(x))$ for some monic prime polynomial $q$ of $F[x]$. As $x^{p^{m}} \in K_{0}, q^{p^{m}} \in K_{0}$ and so $v_{i}^{\prime}\left(q^{p^{m}}\right)=v_{s}\left(q^{p^{m}}\right)>0$. Therefore there exists a nonzero $T_{i}$-topological nilpotent contained in $F[x]$. Moreover, $F$ is a $T_{l}$-bounded set. Indeed, if $B$ is any $T_{l}$-bounded neighborhood of zero, then there exists $k>0$ such that $q^{k} F \subseteq B \cap K_{0}$ as $F$ is bounded for $T_{v_{i}^{\prime}}$. Then $F \subseteq q^{-k} B$, a $T_{i}$-bounded set. Thus by Corollary $1, T_{l}$ is the supremum of a finite family of valuation topologies, each of which is discrete on $F$.

Suppose $s=\infty$. The above argument with $q$ replaced by $x^{-1}$ yields that $T_{l}$ is a Hausdorff, locally bounded topology on $F\left(x^{-1}\right)$ for which there exists a nonzero topological nilpotent $f$ in $F\left[x^{-1}\right]$ and for which $F$ is a $T_{l}$-bounded set. Therefore $T_{i}$ is the supremum of a finite family of valuation topologies having the desired properties.

Corollary 3. Let $F$ be a field, let $x$ be a transcendental element over $F$ and let $L$ be a separable finite algebraic extension of $F(x)$. If $T$ is a Hausdorff locally bounded topology on $L$ for which $F$ is a bounded set and for which there exists a nonzero topological nilpotent, then there exists a finite family $\left\{v_{1}, v_{2}, \ldots, v_{n}\right\}$ of nontrivial valuations on $L$, each of which is trivial on $F$, such that $T=\sup _{1 \leq l \leq n} T_{v_{i}}$.

Proof. Let $y$ be a nonzero topological nilpotent for $T$. By Lemma 3 of [7], $y$ is a transcendental element over $F$ and hence $L$ is a finite algebraic extension of $F(y)$. The proof of Corollary 2 yields that there exists a nonzero $T$-topological nilpotent contained in $F(x)$. Indeed, with the terminology of that proof, let $K_{0}$ be a maximal subfield of $L$ containing $F(y)$ such that $\left.T\right|_{K_{0}}$ has the desired properties and for each $i, 1 \leq i \leq t$, let $v_{i}$ be a nontrivial valuation on $L$ such that $\left.T_{l}\right|_{K_{0}}=\left.T_{v_{i}}\right|_{K_{0}}$. Then $\left.v_{i}\right|_{F(x)}$ is a nontrivial valuation [3, Corollary 2, p. 140] and so there exists a nonzero $z$ in $F(x)$ such that $v_{i}(z) \geq 1$ for $i=1,2, \ldots, t$. If $m \geq 0$ is any integer such that $x^{p^{m}} \in K_{0}$, then $z^{p^{m}} \in K_{0}$ and $z^{p^{m}}$ is a $T_{1}$-topological nilpotent for each $i$. Consequently $z^{p^{m}}$ is a $T$-topological nilpotent. Hence by Corollary $2,\left.T\right|_{F(x)}$ is the supremum of finitely many valuation topologies, each of which is discrete on $F$. Corollary 3 then follows from Theorem 5 of [21].

We note that Corollaries 2 and 3 also follow from a result of Weber [27, Folgerung 4.4].

COROLlary 4. Let $F_{q}$ be a finite field, let $x$ be a transcendental element over $F_{q}$ and let $L$ be a finite algebraic extension of $F_{q}(x)$. If $T$ is a Hausdorff 
locally bounded topology on $L$ for which there exists a nonzero topological nilpotent, then $T$ is the supremum of a finite family of valuation topologies.

Proof. By Theorem 31:9 of [20], there exists a transcendental element $y$ over $F_{q}$ such that $L$ is a finite separable extension of $F_{q}(y)$. The corollary then follows from Corollary 3.

3. Extensions of topologies. Throughout this section, if $p$ is a nonzero proper prime ideal of a Dedekind domain $D$, we denote the locally bounded topologies defined on $D$ and on the quotient field $K$ of $D$ (by the $p$-adic valuation) by $T_{p}$ and $\hat{T}_{p}$ respectively.

Theorem 3. Let $D$ be a Dedekind domain with quotient field $K$. Let $T$ be a Hausdorff locally bounded topology on $D$ for which there exists a nonzero topological nilpotent $x$ in $D$ satisfying:

1. For each neighborhood $V$ of zero, $x V$ is a neighborhood of zero.

2. If $y$ is any nonzero element of $D$ with $D x+D y=D$ and $V$ is any $T$-neighborhood of zero, then there exists $a z$ in $D$ such that $y z-1 \in V$. Then there exists a Hausdorff locally bounded topology $\hat{T}$ on $K$ such that $\left.\hat{T}\right|_{D}=T$. Moreover if $T^{\prime}$ is any Hausdorff locally bounded topology on $K$ for which $\left.T^{\prime}\right|_{D} \subseteq T$, then $T^{\prime} \subseteq \hat{T}$.

Proof. By [25, Theorem 4], there exists a nontrivial norm $N$ on $D$ with $T=T_{N}$. For each $\varepsilon>0$, let $B_{\varepsilon}=\{y \in D: N(y)<\varepsilon\}$. By induction on $n$, $x^{n} B_{1}$ is a $T$-neighborhood of zero for all $n \geq 0$. Replacing $x$ by $x^{m}$ if necessary, we may assume that $N(x)<1$.

For each $\varepsilon>0$, let $\hat{B}_{\varepsilon}=\{y \in K$ : there exist $a$ and $b$ in $D$ with $a$, $b-1 \in B_{\varepsilon}$ and $\left.y=a / b\right\}$. Clearly, $0 \in \hat{B}_{\varepsilon},-\hat{B}_{\varepsilon}=\hat{B}_{\varepsilon}$ and $\hat{B}_{\min \{\varepsilon, \delta\}} \subseteq \hat{B}_{\varepsilon}$ $\cap \hat{B}_{\delta}$ for all $\varepsilon, \delta>0$. So in order to show that $\left\{\hat{B}_{\varepsilon}: \varepsilon>0\right\}$ is a fundamental system of neighborhoods of zero for a ring topology $\hat{T}$ on $K$, it suffices to show that given $\varepsilon>0$ and $y \in K$, there exist positive numbers $\delta, \eta$ and $\gamma$ such that $\hat{B}_{\delta}+\hat{B}_{\delta} \subseteq \hat{B}_{\varepsilon}, \hat{B}_{\eta} \hat{B}_{\eta} \subseteq \hat{B}_{\varepsilon}$ and $y \hat{B}_{\gamma} \subseteq \hat{B}_{\varepsilon}[4$, p. 75].

Let $\varepsilon>0$ and let $\delta>0$ be such that $\left(B_{\delta}+1\right) B_{\delta}+\left(B_{\delta}+1\right) B_{\delta}+B_{\delta}$ $\subseteq B_{\varepsilon}$. If $a, b, c, d \in D$ with $b, d \neq 0$ and $a, c, b-1, d-1 \in B_{\delta}$, then $b d-1=b(d-1)+(b-1) \in\left(B_{\delta}+1\right) B_{\delta}+B_{\delta} \subseteq B_{\varepsilon}$. Furthermore, ad $+b c \in B_{\delta}\left(B_{\delta}+1\right)+\left(B_{\delta}+1\right) B_{\delta} \subseteq B_{\varepsilon}$. So $a / b+c / d \in \hat{B}_{\varepsilon}$ and hence $\hat{B}_{\delta}+\hat{B}_{\delta} \subseteq \hat{B}_{\varepsilon}$.

Let $\varepsilon>0$ and choose $\delta>0$ such that $B_{\delta} B_{\delta}+\left(B_{\delta}+1\right) B_{\delta}+B_{\delta} \subseteq B_{\varepsilon}$. If $a / b, c / d \in \hat{B}_{\delta}$ where $a, c, b-1, d-1 \in B_{\delta}$, then by the above argument, $b d-1 \in B_{\varepsilon}$. Moreover, $a c \in B_{\delta} B_{\delta} \subseteq B_{\varepsilon}$. Hence $\hat{B}_{\delta} \hat{B}_{\delta} \subseteq \hat{B}_{\varepsilon}$. 
Finally let $\varepsilon>0$ and let $f / g \in K$ where $f, g \in D$ and $g \neq 0$. It suffices to show that for $f \neq 0$ and $0<\varepsilon<1$, there exists $\delta>0$ such that $(f / g) \hat{B}_{\delta} \subseteq \hat{B}_{\varepsilon}$. Choose $n>0$ such that $n v_{p}(x)-v_{p}(g) \geq 0$ for all $p$ in $\mathscr{P}$ with $v_{p}(x), v_{p}(g)>0$. Let $c$ be a nonzero element of $D$ such that $v_{p}(c)=0$ for all nonzero proper prime ideals which divide $D x$ and $v_{p}(c) \geq v_{p}(g)$ for all $p$ in $\mathscr{P}$ with $v_{p}\left(x^{n} / g\right)<0$. (The existence of $c$ is guaranteed by [3, Proposition 9, p. 12].) Clearly $D c+D x=D$. Furthermore $c x^{n} / g \in D$. Indeed, if $v_{p}(x)>0$ or if $v_{p}(g)=0$, then $v_{p}\left(c x^{n} / g\right)=$ $v_{p}(c)+v_{p}\left(x^{n} / g\right) \geq 0$. Moreover, if $v_{p}(x)=0$ and $v_{p}(g)>0$, then $v_{p}\left(c x^{n} / g\right)=v_{p}(c)-v_{p}(g) \geq 0$. So $c x^{n}=g d$ for some nonzero $d$ in $D$. As $D c+D x=D$, there exists a nonzero $z$ in $D$ with $c z-1 \in B_{\varepsilon / 2}$. Let $M \geq n$ be such that $x^{M} f z d \in B_{\varepsilon}$ and let $\delta>0$ be such that $B_{\delta}\left(B_{\varepsilon / 2}+1\right)$ $\subseteq B_{\varepsilon / 2}$ and $B_{\delta} \subseteq x^{2 M} B_{1}$. Let $a$ and $b$ be elements of $D$ such that $b \neq 0$ and $a, b-1 \in B_{\delta}$. Then $a=x^{2 M} b_{1}$ for some $b_{1} \in B_{1}$ and so

$$
\frac{f}{g} \frac{a}{b}=\frac{x^{2 M} b_{1} f z d}{g b z d}=\frac{x^{M} x^{s} b_{1} f z d}{b z c}
$$

for some $s \geq 0$. As $N\left(x^{s} b_{1}\right) \leq 1, x^{M} x^{s} b_{1} f z d \in B_{\varepsilon}$. Furthermore by a previous argument, $b z c-1 \in B_{\varepsilon}$ as well. Thus $(f / g) \hat{B}_{\delta} \subseteq \hat{B}_{\varepsilon}$. Consequently, $\left\{\hat{B}_{\varepsilon}: \varepsilon>0\right\}$ is a fundamental system of neighborhoods of zero for a ring topology $\hat{T}$ on $K$.

In order to show that $\hat{T}$ is a locally bounded topology on $K$, it suffices to show that $\hat{B}_{1 / 2}$ is a $\hat{T}$-bounded set. First observe that if $d$ is any element of $D$ with $d-1 \in B_{1 / 2}$, then $D d+D x=D$. Indeed, suppose that $v_{p}(d)$, $v_{p}(x)>0$ for some $p$ in $\mathscr{P}$. Then $v_{p}(d-1)=0$. But $d-1$ is a $T$-topological nilpotent of $D$ and so $(d-1)^{m} \in x B_{1}$ for some $m>0$. Hence $v_{p}(d-1)>0$, a contradiction. Therefore, $D d+D x=D$. Now let $0<\varepsilon$ $<1$ and let $\delta>0$ be such that $\left(B_{\varepsilon / 2}+1\right) B_{\delta} \subseteq B_{\varepsilon / 2}$ and $B_{\delta}\left(B_{N(1)+\varepsilon / 2}\right) \subseteq$ $B_{\varepsilon}$. Let $a, b, c, d \in D$ where $b$ and $d$ are nonzero, $a, b-1 \in B_{\delta}$ and $c, d-1 \in B_{1 / 2}$. By the above remark, there exists a nonzero $y$ in $D$ with $y d-1 \in B_{\varepsilon / 2}$. Hence, $N(y) \leq N(1-d) N(y)+N(y d)<\frac{1}{2} N(y)+$ $(N(1)+\varepsilon / 2)$ and so $N(y)<2(N(1)+\varepsilon / 2)$. Consequently acy $\in$ $B_{\delta}\left(B_{N(1)+\varepsilon / 2}\right) \subseteq B_{\varepsilon}$. Moreover, bdy $-1=(d y)(b-1)+(d y-1) \in$ $\left(B_{\varepsilon / 2}+1\right) B_{\delta}+B_{\varepsilon / 2} \subseteq B_{\varepsilon}$. So $a c / b d \in \hat{B}_{\varepsilon}$ and hence $\hat{B}_{1 / 2}$ is a $\hat{T}$ bounded neighborhood of zero.

We next show that $\left.\hat{T}\right|_{D}=T$. Obviously for any $\varepsilon>0, B_{\varepsilon} \subseteq \hat{B}_{\varepsilon} \cap D$ and so $\left.\hat{T}\right|_{D} \subseteq T$. To prove the reverse inclusion, let $\varepsilon>0$, let $\delta>0$ be such that $\delta \leq \min \{\varepsilon / 2,1 / 2\}$ and let $a / b \in \hat{B}_{\delta} \cap D$ where $a, b-1 \in B_{\delta}$. Denote $a / b$ by $a_{1}$. As $a_{1}=a_{1}(1-b)+a, N\left(a_{1}\right)<N\left(a_{1}\right) \delta+\delta \leq \frac{1}{2} N\left(a_{1}\right)$ $+\delta$. Consequently, $N\left(a_{1}\right)<2 \delta \leq \varepsilon$. Therefore $\hat{B}_{\delta} \cap D \subseteq B_{\varepsilon}$ and hence $\left.\hat{T}\right|_{D}=T$. (We note that as $T$ is Hausdorff, $\hat{T}$ is Hausdorff as well.) 
Finally let $T^{\prime}$ be any Hausdorff locally bounded topology on $K$ for which $\left.T^{\prime}\right|_{D} \subseteq T$. Then $x^{n} \rightarrow 0$ for $T^{\prime}$ and hence by [8, Theorem 6.1], $T^{\prime}$ is normable. Therefore the mapping $y \rightarrow y^{-1}$ is continuous on $K^{*}$ for $T^{\prime}$. (The proof of this assertion is the same as that for normed algebras found on p. 75 of [5].) Let $V$ be any $T^{\prime}$-neighborhood of zero. As $(y, z) \rightarrow y^{-1} z$ is continuous at $(1,0)$ for $T^{\prime}$, there exists a $T^{\prime}$-neighborhood $U$ of zero such that $-1 \notin U$ and $(1+U)^{-1} U \subseteq V$. Let $\delta>0$ be such that $B_{\delta} \subseteq U$ $\cap D$. If $a / b \in \hat{B}_{\delta}$ where $a, b-1 \in B_{\delta}$, then $a \in U$ and $b \in U+1$. So $a / b \in V$ and hence $T^{\prime} \subseteq \hat{T}$.

We note that the topology $\hat{T}$ defined in Theorem 3 is normable as $x^{n} \rightarrow 0$ for $\hat{T}$ and consequently $\hat{T}$ is compatible with the field structure of $K$.

COROLlary. Let $A$ be a principal ideal domain with quotient field $K$ and let $T$ be a Hausdorff locally bounded topology on $A$ for which $A$ is a bounded set. If there exists a nonzero topological nilpotent $x$ in $A$ such that $x V$ is a $T$-neighborhood of zero whenever $V$ is a T-neighborhood of zero, then there exists a locally bounded topology $\hat{T}$ on $K$ with $\left.\hat{T}\right|_{A}=T$.

Proof. Let $y$ be any nonzero element of $A$ such that $A y+A x=A$, let $V$ be any $T$-neighborhood of zero and let $n>0$ be such that $A x^{n} \subseteq V$. Then $A y+A x^{n}=A$ and so there exists $g$ in $A$ with $g y-1 \in A x^{n} \subseteq V$. The corollary then follows from Theorem 3 .

TheOREM 4. Let $D$ be a Dedekind domain with quotient field $K$ such that the class number of $K$ over $D$ is finite and $K \neq D$. Let $T$ be a Hausdorff, nondiscrete, locally bounded topology on $D$. The following are equivalent.

$1^{\circ} D$ is a T-bounded set and there exists a nonzero topological nilpotent $x$ in D such that $x V$ is a T-neighborhood of zero for each T-neighborhood $V$ of zero.

$2^{\circ}$ There exists a sequence $p_{1}, p_{2}, \ldots, p_{n}$ of nonzero proper prime ideals of $D$ such that $T=\sup _{1 \leq i \leq n} T_{p_{i}}$.

$3^{\circ} D$ is a $T$-bounded set, there exists a nonzero topological nilpotent $x$ in $D$ and there exists a locally bounded topology $\hat{T}$ on $K$ such that $\left.\hat{T}\right|_{D}=T$.

$4^{\circ}$ There exists a normable topology $\hat{T}$ on $K$ such that $\left.\hat{T}\right|_{D}=T$ and $D$ is a $\hat{T}$-bounded set. 
Proof. $1^{\circ} \Rightarrow 2^{\circ}$. First notice that as $T$ is Hausdorff and as $D$ is a $T$-bounded set, $x$ is a nonunit of $D$. So $D x=\prod_{i=1}^{n} p_{i}^{\alpha_{i}}$ where $p_{1}, p_{2}, \ldots, p_{n}$ are nonzero proper prime ideals of $D$ and $\alpha_{i} \geq 1$ for $i \in[1, n]$. As before, an inductive argument establishes that $D x^{m}$ is a $T$-neighborhood of zero for $m \geq 0$. Consequently, $\left\{D x^{m}: m \geq 0\right\}$ is a fundamental system of neighborhoods of zero for $T$ and for $\sup _{1 \leq i \leq n} T_{p_{i}}$.

$2^{\circ} \Rightarrow 3^{\circ}$ is obvious.

$3^{\circ} \Rightarrow 4^{\circ}$. As $T$ is Hausdorff, $\hat{T}$ is Hausdorff as well. Also, since $x^{n} \rightarrow 0$ for $T, x^{n} \rightarrow 0$ for $\hat{T}$ and so $\hat{T}$ is normable [8, Theorem 6.1]. Let $\hat{V}$ be any $\hat{T}$-bounded neighborhood of zero and let $n>0$ be such that $x^{n} D \subseteq \hat{V} \cap D$. Then $D \subseteq x^{-n} \hat{V}$, a $\hat{T}$-bounded set. Thus $D$ is a $\hat{T}$-bounded set.

$4^{\circ} \Rightarrow 1^{\circ}$. By Theorem 2, $\hat{T}=\sup _{1 \leq i \leq n} \hat{T}_{p_{i}}$ for some sequence $p_{1}, p_{2}, \ldots, p_{n}$ of nonzero proper prime ideals of $D$. Clearly, $D$ is a $T$ bounded set. As the class number of $K$ over $D$ is finite, there exists a nonzero element $x$ in $D$ with $v_{p_{1}}(x)>0$ for $i=1,2, \ldots, n$ and $v_{p}(x)=0$ for $p$ in $\mathscr{P} \backslash\left\{p_{1}, p_{2}, \ldots, p_{n}\right\}$. Then $x$ is a $\hat{T}$-topological nilpotent and hence a $T$-topological nilpotent. Moreover for any $m \geq 0, x(\{y \in K$ : $v_{p_{i}}(y) \geq m$ for $\left.\left.i \in[1, n]\right\} \cap D\right) \supseteq\left\{y \in D: v_{p_{i}}(y) \geq m+v_{p_{i}}(x)\right.$ for $i \in[1, n]\}$. So $x V$ is a $T$-neighborhood of zero for each $T$-neighborhood $V$ of zero.

COROLlary 1. If $A$ is a principal ideal domain which is not a field and if $T$ is a nondiscrete normable topology on $A$ for which $A$ is a bounded set, then there exists a locally bounded topology on the quotient field of $A$ whose restriction to $A$ is $T$ if and only if $T$ is the supremum of a finite family of p-adic topologies.

In [7] we characterized the nondiscrete normable topologies on the ring of integers $Z$ as follows. For any prime ideal $p$ and for any positive integer $n,\left\{p^{n}\right\}$ is a fundamental system of neighborhoods of zero for a locally bounded topology $T_{p^{n}}^{\prime}$ on $Z$. If $T$ is a nondiscrete normable topology on $Z$, then there exist disjoint finite subsets $\mathscr{P}_{1}$ and $\mathscr{P}_{2}$ of $\mathscr{P}$ and positive integers $n(p)$ for each $p$ in $\mathscr{P}_{2}$ such that $T=\sup \left(\sup _{p \in \mathscr{P}_{1}} T_{p}\right.$, $\sup _{p \in \mathscr{P}_{2}} T_{\mathrm{p}^{n(p)}}^{\prime}$ ). (See also [17] and [19].) The analogous characterization of the nondiscrete normable topologies on the polynomial ring $F[x]$ for which $F$ is bounded was also given in [7]. The next two corollaries describe 
the extendable nondiscrete normable topologies on $Z$ and on $F[x]$ for which $F$ is bounded.

COROllary 2. If $N$ is a nontrivial norm on $Z$ and $T$ is the topology defined by $N$, then there exists a locally bounded topology $\hat{T}$ on the rational field with $\left.\hat{T}\right|_{Z}=T$ if and only if $T$ is the supremum of a finite family of p-adic topologies.

Proof. If $N$ is a nontrivial norm on $Z$, then there exists $m>1$ such that $N(m)<1$. Let $M=\max \{N(i):-m<i<m\}$ and let $x \in Z$. Then $x=\sum_{i=0}^{n} a_{i} m^{i}$ where $-m<a_{i}<m$ for $i \in[0, n]$. Hence

$$
N(x) \leq M \sum_{i=0}^{n}[N(m)]^{i} \leq M \sum_{i=0}^{\infty}[N(m)]^{i}=M \frac{1}{1-N(m)} .
$$

Therefore $Z$ is bounded in norm and so the corollary follows from Corollary 1.

COROllary 3. Let $F$ be a field and let $x$ be a transcendental element over $F$. If $N$ is a nontrivial norm on $F[x]$ for which $F$ is norm-bounded, then there exists a locally bounded topology $\hat{T}$ on $F(x)$ whose restriction to $F[x]$ is the topology $T$ defined by $N$ if and only if $T$ is the supremum of a finite family of p-adic topologies.

Proof. We observe that as $T$ is Hausdorff and $F$ is bounded in norm, for each nonzero $y$ in $F[x]$ with $N(y)<1$, deg $y \geq 1$. Corollary 3 follows from this observation and an argument similar to the one used in the proof of Corollary 2.

We conclude this section by observing that an appropriate modification of the proof of Theorem 3 yields the following result.

THEOREM 5. Let $I$ be a unique factorization domain and let $K$ be the quotient field of $I$. Let $T$ be a Hausdorff, locally bounded topology on I for which there exists a nonzero topological nilpotent $x$ in I satisfying:

1. For each neighborhood $V$ of zero, $x V$ is a neighborhood of zero.

2. If $y$ is any nonzero element of $I$ with $(x, y)=1$ and $V$ is any neighborhood of zero, then there exists a $z$ in I with $y z-1 \in V$.

Then there exists a Hausdorff, locally bounded topology $\hat{T}$ on $K$ whose restriction to $I$ is $T$.

4. Locally compact Dedekind domains. In [23] Warner raised the question of whether the topology of a compact integral domain $I$ can be 
extended to the quotient field of $I$. In [22] he showed that a compact Dedekind domain $D$ is a local principal ideal domain for which $\left\{m^{n}\right.$ : $n \geq 0\}$ is a fundamental system of neighborhoods of zero where $m$ is the unique maximal ideal of $D$ (Theorems 1 and 3). Thus a compact Dedekind domain $D$ is a locally compact bounded ring whose given topology is the restriction to $D$ of the $m$-adic topology defined on the quotient field of $D$. We show conversely that if $D$ is a locally compact bounded Dedekind domain and if there exists a ring topology on the quotient field of $D$ whose restriction to $D$ is the given topology, then $D$ is a compact principal ideal domain. Moreover, if $D$ is a Dedekind domain with quotient field $K$ such that the class number of $K$ over $D$ is finite, then $D$ is locally compact and its given topology extends to a ring topology on $K$ if and only if $D$ is a compact principal ideal domain.

TheOREM 6. Let $D$ be a Dedekind domain which is not a field, let $K$ be the quotient field of $D$ and let $T$ be a nondiscrete Hausdorff ring topology on $D$. The following are equivalent.

$1^{\circ}(D, T)$ is locally compact, there exists a nonzero proper prime ideal $p$ of $D$ such that $p^{n}$ is a T-neighborhood of zero for all $n \geq 0$ and there exists a ring topology $\hat{T}$ on $K$ with $\left.\hat{T}\right|_{D}=T$.

$2^{\circ} \mathrm{D}$ is a compact principal ideal domain.

Proof. by the previous remarks, it suffices to show that $1^{\circ}$ implies $2^{\circ}$. As $D$ is not a field, $D$ is totally disconnected [24, Theorem 2] and hence there exists a compact open subring $B$ of $D$ such that $\operatorname{Rad} B$ is a compact open subring of $D$ contained in $p$ [16, Lemma 4 and 24, Theorem 3]. As $\cap_{n=1}^{\infty} p^{n}=(0),\left\{B \cap p^{n}: n \geq 0\right\}$ is a fundamental system of neighborhoods of zero for a Hausdorff topology $T^{\prime}$ on $B$ weaker than $\left.T\right|_{B}$. Hence $T^{\prime}=\left.T\right|_{B}$ and so $\left\{B \cap p^{n}: n \geq 0\right\}$ is a fundamental system of neighborhoods of zero for $T$.

Let $a$ be a nonzero element of $\operatorname{Rad} B$. Then $D a=p^{\alpha_{0}} \prod_{i=1}^{n} p_{i}^{\alpha_{t}}$ where $p_{1}, p_{2}, \ldots, p_{n}$ are nonzero proper prime ideals of $D$ and $\alpha_{0}, \alpha_{1}, \ldots, \alpha_{n}$ are positive integers. We first show that $p \prod_{i=1}^{n} p_{i}$ is a $T$-topologically nil ideal of $D$, that is, if $b \in p \prod_{i=1}^{n} p_{i}$, then $b^{m} \rightarrow 0$ for $T$.

Let $b \in p \prod_{i=1}^{n} p_{i}$, let $m \geq 1$ be such that $b^{m} / a \in D$ and let $t \geq 1$ be such that $\left(b^{m} / a\right)\left(B \cap p^{t}\right) \subseteq B$. Then $\left(b^{m} / a\right)\left(B \cap p^{t}\right) \subseteq B \cap p^{t}$ and so for all $s \geq 1,\left(b^{m} / a\right)^{s}\left(B \cap p^{t}\right) \subseteq B \cap p^{t}$. In particular, $b^{m t}=\left(b^{m} / a\right)^{t} a^{t}$ $\in B \cap p^{t}$. Consequently for any $N_{0} \geq 1$ and any $N \geq N_{0},\left(b^{m t}\right)^{N} \in B \cap$ $p^{t N} \subseteq B \cap p^{N_{0}}$. Therefore, $b^{m t}$ is a $T$-topological nilpotent. As $\left\{b^{r}: 1 \leq r\right.$ $<m t\}$ is a $T$-bounded set, it follows that $b$ is a $T$-topological nilpotent. So 
$p \prod_{i=1}^{n} p_{i}$ is a $T$-topologically nil ideal of $D$. By [15, Corollary to Theorem 12], $p \prod_{i=1}^{n} p_{i} \subseteq \operatorname{Rad} D$. Hence if $q$ is any nonzero proper prime ideal of $D$, then $p \prod_{l=1}^{n} p_{i} \subseteq q$ and so $q \in\left\{p, p_{1}, \ldots, p_{n}\right\}$. Therefore, $D$ is a principal ideal domain [28, Theorem 16, p. 278]. As $p^{n}$ is a $T$-neighborhood of zero for all $n \geq 0$, the proof of Theorem 6 of [24] yields that $D$ has only one maximal ideal $D c$, all the nonzero ideals of $D$ are $T$-open and $D c$ is a $T$-topologically nil ideal.

We next show that if $V$ is any $T$-neighborhood of zero, then $c V$ is a $T$-neighborhood of zero. As $p=D c$ is a $T$-neighborhood of zero, we may assume that $V \subseteq p$. Let $\hat{V}$ and $\hat{W}$ be $\hat{T}$-neighborhoods of zero with $\hat{V} \cap D \subseteq V$ and $\hat{W} \cup c \hat{W} \subseteq \hat{V}$. Suppose $y \in \hat{W}$ and $c y \in D$. Then $c y \in$ $\hat{V} \cap D \subseteq p$ and so $v_{p}(c y) \geq 1$. Hence $v_{p}(y) \geq 0$, that is, $y \in D$. Consequently $(c \hat{W}) \cap D \subseteq c(\hat{W} \cap D) \subseteq c V$. Therefore $c V$ is a $T$-neighborhood of zero whenever $V$ is a $T$-neighborhood of zero. It follows by induction that for any $n \geq 0$ and any $T$-neighborhood $V$ of zero, $c^{n} V$ is a $T$-neighborhood of zero.

Now let $a$ be any nonzero element of $D$ and let $V$ be any $T$-neighborhood of zero. Then $a=b c^{n}$ for some $n \geq 0$ and some unit $b$ of $D$. By the above remarks, $a V=b c^{n} V$ is a $T$-neighborhood of zero. Hence by [16, Theorems 8 and 23, Theorems 5 and 7], $D$ is a compact ring.

THEOREM 7. Let $D$ be a Dedekind domain which is not a field, let $K$ be the quotient field of $D$ and let $T$ be a nondiscrete Hausdorff ring topology on $D$. If $(D, T)$ is locally compact, then the following are equivalent.

$1^{\circ} D$ is a T-bounded set and there exists a locally bounded ring topology $\hat{T}$ on $K$ such that $\left.\hat{T}\right|_{D}=T$.

$2^{\circ} \quad D$ is a T-bounded set and there exists a field topology $\hat{T}$ on $K$ such that $\left.\hat{T}\right|_{D}=T$.

$3^{\circ} D$ is a $T$-bounded set and there exists a ring topology $\hat{T}$ on $K$ such that $\left.\hat{T}\right|_{D}=T$.

$4^{\circ} D$ is a compact principal ideal domain.

Proof. Clearly $2^{\circ}$ implies $3^{\circ}$ and as before, $4^{\circ}$ implies $1^{\circ}$ follows from Theorems 1 and 3 of [22]. By [15, Theorem 14], if $B$ is any compact open subring of $D$, then $\operatorname{Rad} B$ is a $T$-topologically nil ideal of $B$ and hence is a $\hat{T}$-topologically nil ideal of $B$ for any ring topology $\hat{T}$ on $K$ with $\left.\hat{T}\right|_{D}=T$. Thus if $1^{\circ}$ holds, then $\hat{T}$ is normable [8, Theorem 6.1]. Consequently $\hat{T}$ is a field topology on $K$. So $1^{\circ}$ implies $2^{\circ}$.

By Theorem 6, in order to show that $3^{\circ}$ implies $4^{\circ}$, it suffices to show that there exists a nonzero proper prime ideal $p$ of $D$ such that $p^{n}$ is a 
$T$-neighborhood of zero for all $n \geq 0$. By [12, Lemma 4.5], $D$ contains a proper $T$-open ideal and hence a nonzero, proper, $T$-open prime ideal $p$.

First observe that $D$ is a $\hat{T}$-bounded set. Indeed, if $B$ is any compact $T$-open subring of $D$, then there exists a nonzero $b$ in $B$ such that $D b \subseteq B$. As $\left.\hat{T}\right|_{D}=T, B b^{-1}$ is a $\hat{T}$-compact subset of $K$. Therefore $D$ is a $\hat{T}$-bounded subset of $K$. Now let $\hat{V}$ and $\hat{W}$ be $\hat{T}$-neighborhoods of zero such that $\hat{V} \cap D \subseteq p$ and $D \hat{W} \subseteq \hat{V}$. Suppose there exists a $y$ in $\hat{W}$ with $v_{p}(y)<0$. Let $x$ be a nonzero element of $D$ such that $x y \in D$ and $v_{p}(x)=-v_{p}(y)$. Then $x y \in \hat{V} \cap D \subseteq p$ but $v_{p}(x y)=0$, a contradiction. Thus for all $y$ in $\hat{W}, v_{p}(y) \geq 0$. Consequently if $c$ is any nonzero element of $D$ with $v_{p}(c) \geq 1$, then $\left(c^{n} \hat{W}\right) \cap D \subseteq p^{n}$ for all $n \geq 0$. So $p^{n}$ is a $T$-neighborhood of zero for all $n \geq 0$.

THEOREM 8. Let $D$ be a Dedekind domain which is not a field, let $K$ be the quotient field of $D$ and let $T$ be a Hausdorff, nondiscrete ring topology on $D$ such that $(D, T)$ is locally compact. If the class number $\alpha$ of $K$ over $D$ if finite, then the following statements are equivalent.

$1^{\circ}$ There exists a locally bounded ring topology $\hat{T}$ on $K$ such that $\left.\hat{T}\right|_{D}=T$.

$2^{\circ}$ There exists a field topology $\hat{T}$ on $K$ such that $\left.\hat{T}\right|_{D}=T$.

$3^{\circ}$ There exists a ring topology $\hat{T}$ on $K$ such that $\left.\hat{T}\right|_{D}=T$.

$4^{\circ} D$ is a compact principal ideal domain.

Proof. We show that $3^{\circ}$ implies $4^{\circ}$. Let $p$ be a nonzero, proper, $T$-open, prime ideal of $D$ and let $c$ be a nonzero element of $D$ with $p^{\alpha}=D c$. We first show that $c V$ is a $T$-neighborhood of zero for any $T$-neighborhood $V$ of zero. As before, we may assume that $V \subseteq p$. Let $\hat{V}$, $\hat{W}$ and $\hat{U}$ be $\hat{T}$-neighborhoods of zero such that $\hat{V} \cap D \subseteq V, \hat{W} \cup(\hat{W})^{\alpha}$ $\subseteq \hat{V}$ and $c \hat{U} \subseteq \hat{W}$. Suppose $y \in \hat{W} \cap \hat{U}$ and $c y \in D$. Then for all $q$ in $\mathscr{P} \backslash\{p\}, v_{q}(y) \geq 0$. Suppose that $y \notin D$, that is, $v_{p}(y)<0$. As $c y \in D$, $\alpha=v_{p}(c)=-v_{p}(y)+n$ for some $n \geq 0$. Then $c^{-v_{p}(y)} y^{\alpha}=(c y)^{-v_{p}(y)} y^{n}$ $\in(\hat{W})^{\alpha} \subseteq \hat{V}$. Furthermore as $v_{p}\left(c^{-v_{p}(y)} y^{\alpha}\right)=0, c^{-v_{p}(y)} y^{\alpha} \in D$. So $c^{-v_{p}(y)} y^{\alpha} \in \hat{V} \cap D \subseteq p$, a contradiction. Therefore $c(\hat{W} \cap \hat{U}) \cap D$ $\subseteq c(\hat{W} \cap \hat{U} \cap D) \subseteq c(\hat{V} \cap D) \subseteq c V$ and so $c V$ is a $T$-neighborhood of zero. Consequently for all $n \geq 0, p^{n}$ contains $D c^{n}$, a $T$-neighborhood of zero. Thus by Theorem $6,3^{\circ}$ implies $4^{\circ}$.

In [24], Warner proved that there exists a locally compact principal ideal domain which is not compact (Theorems 15 and 21). Thus by 
Theorem 8, there exists a locally compact prinicpal ideal domain $A$ whose given topology does not extend to a ring topology on the quotient field of A.

\section{REFERENCES}

[1] J. M. Anthony, Topologies for quotient fields of commutative integral domains, Pacific J. Math., 36 (1971), 585-601.

[2] V. I. Arnautov, Continuation of a topology of the commutative ring to some of its quotient rings, (Russian), Mat. Issled. vyp. 48 (1978), Algebra i moduli, 3-13.

[3] N. Bourbaki, Algèbre Commutative, Ch. 7, Paris, Hermann, 1965.

[4] _ Topologie Générale, Ch. 3-4, Hermann, 1960.

[5] _ Topologie Générale, Ch. 9, Paris, Hermann, 1958.

[6] J. Cohen, Locally bounded topologies on $F(X)$, Pacific J. Math., 70 (1977), 125-132.

[7] , Topologies on the ring of integers of a global field, Pacific J. Math., 93 (1981), 269-276.

[8] P. M. Cohn, An invariant characterization of pseudo-valuations on a field, Proc. Cambridge Phil. Soc., 50 (1954), 159-177.

[9] E. Correl, Topologies on quotient fields, Duke Math. J., 35 (1968), 175-178.

[10] M. Endo, On the embedding of topological rings into quotient rings I, J. Fac. Sci. Univ. Tokyo, Sec. I 10 (1963), 196-214.

[11] B. Gelbaum, G. Kalisch and J. Olmsted, On the embedding of topological semigroups and integral domains, Proc. Amer. Math. Soc., 2 (1951), 805-821.

[12] O. Goldman and Chih-Han Sah, On a special class of locally compact rings, J. Algebra, 4 (1966), 71-95.

[13] J. Heine and S. Warner, Ring topologies on the quotient field of a Dedekind domain, Duke Math. J., 40 (1973), 473-486.

[14] A. Jebli, Sur certaines topologies lineaires, Publ. Sem. Math. Univ. Rennes-Colloq. d'Algèbre Commutative (1972), 7.1-7.5.

[15] I. Kaplansky, Topological rings, Amer. J. Math., 69 (1947), 153-183.

[16] __ Locally compact rings, Amer. J. Math., 70 (1948), 447-459.

[17] F. I. Karpelevic, Pseudonorms in the ring of integers (Russian), Uspehi Mat. Nauk 3 vyp., 5 (1948), 174-177.

[18] K. Kiyek, Pseudobetragsfunktionen auf Quotientenkörpern von Dedekindringen, J. Reine Angew. Math., 274 / 275 (1975), 244-257.

[19] K. Mahler, Über pseudobewertungen III, Acta. Math., 67 (1936), 283-328.

[20] O. T. O'Meara, Introduction to Quadratic Forms, Berlin, Springer-Verlag, 1963.

[21] T. Rigo and S. Warner, Topologies extending valuations, Canad. J. Math., 30 (1978), 164-169.

[22] S. Warner, Compact noetherian rings, Math. Ann., 141 (1960), 161-170.

[23] _ Compact rings, Math. Ann., 145 (1962), 52-63.

[24] _ Locally compact principal ideal domains, Math. Ann., 188 (1970), 317-334.

[25] Normability of certain topological rings, Proc. Amer. Math. Soc., 33 (1972), 423-427.

[26] H. Weber, Charakterisierung der lokalbeschränkten Ringtopologien auf globalen Körpern, Math. Ann., 239 (1979), 193-205.

[27] _ Topologische Charakterisierung globaler Körper und algebraischer Funktionenkörper in einer Variablen, Math. Z., 169 (1979), 167-177. 
[28] O. Zariski and P. Samuel, Commutative Algebra, Vol. I., Princeton, Van Nostrand, 1958.

Received August 4, 1982 and in revised form January 12, 1984.

North Carolina State University

RALEIGH, NC 27650 



\section{PACIFIC JOURNAL OF MATHEMATICS EDITORS}

DONALD BABBITT (Managing Editor)
University of California
Los Angeles, CA 90024
CHARLES R. DePrima
California Institute of Technology
Pasadena, CA 91125
R. FinN
Stanford University
Stanford, CA 94305

HERMANN FLASCHKA

University of Arizona

Tucson, AZ 85721

RAMESH A. GANGOLLI

University of Washington

Seattle, WA 98195

ROBION KIRBY

University of California

Berkeley, CA 94720

C. C. MOore

University of California

Berkeley, CA 94720
Hugo RossI

University of Utah

Salt Lake City, UT 84112

H. SAMELSON

Stanford University

Stanford, CA 94305

HaRold STARK

University of California, San Diego

La Jolla, CA 92093

\section{ASSOCIATE EDITORS}
R. ARENS
E. F. BECKENBACH (1906-1982)
B. H. NEUMANN
F. WOLF
K. YoshidA

\section{SUPPORTING INSTITUTIONS}

UNIVERSITY OF ARIZONA

UNIVERSITY OF BRITISH COLUMBIA

CALIFORNIA INSTITUTE OF TECHNOLOGY

UNIVERSITY OF CALIFORNIA

MONTANA STATE UNIVERSITY

UNIVERSITY OF NEVADA, RENO

NEW MEXICO STATE UNIVERSITY

OREGON STATE UNIVERSITY
UNIVERSITY OF OREGON

UNIVERSITY OF SOUTHERN CALIFORNIA

STANFORD UNIVERSITY

UNIVERSITY OF HAWAII

UNIVERSITY OF TOKYO

UNIVERSITY OF UTAH

WASHINGTON STATE UNIVERSITY

UNIVERSITY OF WASHINGTON 


\section{Pacific Journal of Mathematics}

\section{Vol. 117, No. $1 \quad$ January, 1985}

Amos Altshuler and Leon Steinberg, The complete enumeration of the

4-polytopes and 3 -spheres with eight vertices $\ldots \ldots \ldots \ldots \ldots \ldots \ldots \ldots$

Michael James Beeson, The $6 \pi$ theorem about minimal surfaces . . . . . . . . 17

Jeffrey Lawrence Caruso and Stefan Waner, An approximation theorem

for equivariant loop spaces in the compact Lie case ...............27

Jo-Ann Deborah Cohen, Topologies on the quotient field of a Dedekind

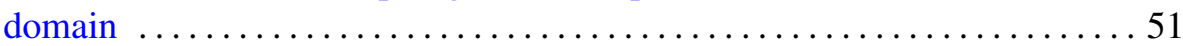

Szymon Dolecki, Gabriele H. Greco and Alois Andreas Lechicki,

Compactoid and compact filters .........................6 69

Roger William Hansell (Sr.), Generalized quotient maps that are

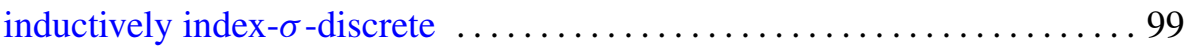

Gerhard Huisken, Capillary surfaces over obstacles $\ldots \ldots \ldots \ldots \ldots \ldots \ldots 121$

Jun Shung Hwang, A problem on continuous and periodic functions . . . . 143

Ronald Fred Levy and Michael David Rice, The extension of equi-uniformly continuous families of mappings $\ldots \ldots \ldots \ldots \ldots \ldots 149$

Kevin Mor McCrimmon, Derivations and Cayley derivations of generalized

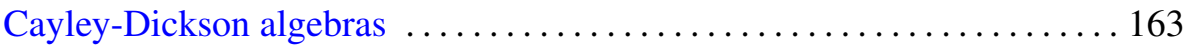

H. M. (Hari Mohan) Srivastava, A multilinear generating function for the Konhauser sets of biorthogonal polynomials suggested by the Laguerre

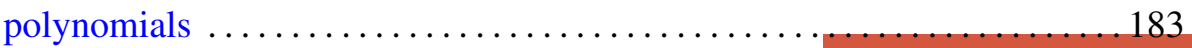

Zhu Jia Lu, Some maximum properties for a family of singular hyperbolic operators 